\title{
Commentary
}

\section{mHealth intervention and health-related quality of life for haemodialysis patients in selected counties of Kenya}

\author{
Martin Muchangi Josphat $^{1 *}$, John Kariuki ${ }^{1}$, George Kimathi ${ }^{2}$
}

\author{
${ }^{1}$ Mount Kenya University, School of Public Health, Kenya \\ ${ }^{2}$ Amref International University, School of Public Health, Kenya
}

Received: 21 January 2022

Revised: 18 February 2022

Accepted: 19 February 2022

\section{*Correspondence:}

Martin Muchangi Josphat,

E-mail: mmchangi@yahoo.com

Copyright: $@$ the author(s), publisher and licensee Medip Academy. This is an open-access article distributed under the terms of the Creative Commons Attribution Non-Commercial License, which permits unrestricted non-commercial use, distribution, and reproduction in any medium, provided the original work is properly cited.

\section{INTRODUCTION}

Chronic kidney disease (CKD) is a condition in which the kidneys have lost functions of filtering blood optimally leading to excess fluid and waste in blood with associated health complications. ${ }^{1}$ the disease is characterised by more than three months loss of kidney functions based on the presence of pathological abnormalities and/or elevated markers of kidney dysfunction. ${ }^{2}$ if not reversed, CKD may progress to absolute kidney failure also known as end-stage renal disease (ESRD). Patients ESRD require regular maintenance dialysis or kidney transplant for the patient to survive. ${ }^{3}$ according to, ESRD is one of the major causes of mortality, and impaired qol. ${ }^{4}$

Ranked $12^{\text {th }}$ most dominant cause of death globally, CKD is associated with over 1.1 million deaths annually hence presenting a major public health challenge. ${ }^{5}$ The prevalence of CKD is increasing alarmingly and conformation, the prevalence of diabetes related kidney disease increased by remarkable margin of $39.5 \%$ between the year 2005 and 2015. ${ }^{6}$ In sub Saharan Africa (SSA), albeit poorly documented, the CKD prevalence is estimated at $13.9 \% .^{7}$ This is higher than HIV prevalence in the region which was estimated at $4 \%$ in the year $2017 .{ }^{8}$ As projected with other Non-communicable diseases, the increased prevalence of CKD and ESRD will affect developing countries, especially in the SSA countries, where the risk factors are highly exacerbated by poverty and weak health systems. ${ }^{9}$ ESRD has major negative and complex implications to social and economic structures to the affected populations as well as countries' economies and therefore needs focused attention. ${ }^{10}$

Although CKD prevalence in Kenya is not clearly known, it is estimated that $13.9 \%$ of the population suffer the disease which is equivalent to SSA estimates and nearly that of Tanzania which standing at $13.6 \% .^{11}$ Kenya like other developing countries has recorded increased CKD prevalence secondary to changing risk factors such as lifestyle and demographic shift. Of note is the epidemiologic changes with regards to lifestyle related diseases that include hypertension diabetes among the rural and urban populations largely correlated with CKD. ${ }^{12}$

To address the challenges of equipment deficits in public health facilities and the ever increasing need for dialysis, the government of Kenya (GoK) initiated a flagship program to upgrade hospitals countrywide to provide specialized medical equipment through the managed equipment service (MES) program. ${ }^{13}$ This significantly increased access to haemodialysis in the peripheral health facilities and offered opportunities for rural populations to access haemodialysis which was initially concentrate in city and urban hospitals. ${ }^{14}$

Positive clinical outcome among dialysis patients largely depends on adherence to treatment regimens and instructions provided by the health care provider. Clinical outcomes consequent to adherence or non-adherence have undisputable influence on QoL. ${ }^{15}$ According to the World Health Organisation (WHO), health related QoL (HRQOL) encompasses various dimensions including 
physical, mental, and social functioning. Based on experts view, HRQOL measurements transcend epidemiologic measurements such as morbidity and mortality. ${ }^{16}$ It's also notable that HRQOL is affected by many factors, key among them; social demographic, economic, and social relationship factors. ${ }^{4}$ Patients in Kenya like those in many other African countries suffer low awareness on CKD and therefore present to diagnostic centres with terminal complications. ${ }^{17}$ Poor awareness and misconceptions on CKD complicates patient care and may worsen the overall clinical outcomes. ${ }^{18}$ It is largely agreed that targeted educational intervention is associated with improvements in knowledge, adherence and QoL among dialysis patients. ${ }^{19}$

As defined by the, mHealth is a component of electronic health (eHealth) which involves advancing health services using different types of electronic platforms. mHealth has multiple benefits key among them: convenience, improved speed of information passage, reduced workload and paper-work and reduced transaction costs. ${ }^{20,21}$ Evidence regarding potential benefits of mHealth such as improved adherence and improved medical outcomes is developing as shown in previous studies that were conducted in over 30 LMICs. ${ }^{22}$

The rapid growth of use mobile of phone and associated technology including wide network coverage in Kenya provides great potential for improving health care delivery systems but this potential has not been fully exploited. ${ }^{23}$ Patient-focused support and management through mobile phone-based platforms can provide targeted information aimed at behaviour improvement such as adherence to haemodialysis and its treatment modalities. One component of mHealth involves the use of SMSs which is a hoard and forward platform and can be stored in the mobile phone for future reference. The WHO postulates that short message service (SMS) can increase demand among the clients and improve health services in general. ${ }^{24}$ Despite clear and foreseeable benefits of mHealth, scanty information is available on effectiveness of mobile phone SMS in improving adherence to haemodialysis and HRQOL in Kenya. The study therefore seeks to establish the effects of using mobile SMSs to improve adherence and HRQOL for haemodialysis patients.

\section{Objectives}

The general objective to study the effect of mHealth interventions on HRQOL for haemodialysis patients in selected counties of Kenya. The specific objectives will be to: (1) To study the effects of mHealth on selfmanagement knowledge, (2) adherence to haemodialysis treatment (3) stress management (4) on life style change and (5) the combined effect of mHealth on HRQOL of haemodialysis patients.

\section{Conceptual framework}

Health education and follow ups for haemodialysis patients are one of the key interventions for appropriate adherence and effective treatment of haemodialysis patients. The main focus of this quasi-experimental study will be to evaluate the efficacy of mHealth based health education on adherence to haemodialysis treatment and HRQOL for haemodialysis patients in Central and Eastern region counties of Kenya. The independent variables in this study are divide into two namely patient factors and health system and access factors. The patient factors shall include: Age, sex, education level, marital status, income, social economic status, occupation, religion and commodities while health system and access factors shall include: Distance to dialysis centre, frequency of haemodialysis, dialysis equipment and functionality uptime. The study also will consider other subsidiary independent variables such as mobile phone ownership, ability to read phone messages. Since this study shall have both the intervention and the control groups, mHealth based education is considered as an intervening variable. Additionally, adherence to haemodialysis services which may result from reminders or motivation through health education is considered as a modifier to the dependent variable. Lastly, the study considers the HRQOL as the dependent variable and shall be measured through physical, mental, emotional and social functioning scores. The study will strive to establish the extent to which health education and follow up modifies such behaviour and whether there is inherent advantage of stepping up such interventions to improve management of ESRD patients. The study will thereafter measure health related QoL comparing the intervention and the non-intervention groups.

\section{METHODS}

This study will be carried out from hospitals located in Embu, Meru, Nyeri, Kirinyaga, and Kiambu counties of Kenya. Intervention site hospitals will be sites will be Embu PG hospital, (Embu), Nyeri PG Hospital (Nyeri), Gatundu DH, Thika level 5 hospital (Kiambu) while control site hospitals are Meru referral hospital (Meru), Kerugoya referral Hospital (Kirinyaga) and Gatundu hospital (Kiambu). The key elements of the mHealth intervention will include developing health education messages aimed at; (1) enhancing self-management on haemodialysis patients; (2) lifestyle change; (3) adherence to medication and; (4) promoting stress management. The main activities of mHealth intervention will involve: (1) developing a mHealth platform and loading the health education message into the platform and; (2) disseminating health education information to all haemodialysis patients in the intervention site. The research design will be a quasi-experimental adopting a pre-test and post post-test study design. A preintervention survey will be conducted before the intervention from a sample of haemodialysis patients in both intervention and control sites. Haemodialysis patients in control site will receive standard treatment procedure provided in government hospitals across Kenya. A post intervention survey will be conducted from a sample of haemodialysis patients in both intervention and control sites. The study population will be haemodialysis patients seeking dialysis treatment in 
various government managed dialysis centres in Eastern and Central region counties of Kenya. Data from the hospital records indicate that the total number of patients with CKD in both intervention and control sites is 126 . This is pegged on hospital capacities in the six hospitals selected in the study area. The table below shows the distribution of patients in the six hospitals selected in this study.

The dependent variables include; (i) self-management knowledge among haemodialysis patients (knowledge on acceptable fluids intake, and knowledge on the nutrition and dietetic measures to adhere to), (ii) knowledge on adherence to medication among haemodialysis patients, (iii) stress management among haemodialysis patients, (iv) Lifestyle change among haemodialysis patients (v) and health related of QoL (HROQL) among haemodialysis patients.

All CKD patients on chronic haemodialysis treatment will be included in the study. For ethical reasons, only adult participants will be recruited. The study will also restrict itself to patients attending selected health facilities in intervention and control sites. The study will deliberately exclude any CKD patient with any physical, mental or physiological strain (Patients with cognitive, poor mental dexterity or psychological disorder) that could restrain him/her in participating in the study. Expectant women will also be excluded in the study due to the potential physiological burden. Since the study will use mobile phones for messaging, patients without phones or who fail to consent to mobile phone messaging will be excluded from the study.

The study will collect data using a variety of forms (WHO standardised and un-standardised) that will be uploaded into the open data kit (ODK) platform. Two surveys will be conducted; a pre-intervention and postintervention survey. In regard to data management and processing, data in the ODK platform will be exported to excel for cleaning and to SPSS for analysis. Descriptive statistics will be used to describe different variables in the study. Effect of mHealth on the outcome variables (Knowledge on self-management, adherence to treatment, stress management, lifestyle change and health related QoL) will be measured using paired $\mathrm{T}$ tests, difference in differences (DiDs), and Odds ratios. This study will employ WHO validated questionnaires in all variables that have validated data collection tools such as stress management and HRQOL. Any other data collection tool will be subjected to validation through seeking expert opinions and inputs. Reliability of all data collection tools will be tested using Cronbach alpa statistic in the piloting stage of this study. Any sets of questions that don't meet the 0.7 to 0.9 reliability index threshold will be revised. Research assistants will be recruited, trained, and tested for competency in administering the questionnaires. Only those will the required competency will be retained for the data collection exercise.

\section{CONCLUSION}

The absence of systems to enhance medical education, offer psychological support and promote adherence to haemodialysis treatment highlights the need to develop simple, cost effective and convenient mhealth platforms that can be integrated into the overall Kenya national kidney health programme. As per the main objective, this study will facilitate the understanding of the effects of mHealth on HRQoL. The findings will redound to development of large scale mHealth based medical education platform for haemodialysis patients with potential for scale as a critical public health intervention. In the academic space, the study will fill the knowledge gap of mHealth influences on HRQoL for haemodialysis patients. It is envisaged that the study will demonstrate ways and methods of conducting similar studies in future. Lastly, the study findings seek to provide basis for further research on behaviour interventions to improve HRQoL.

\section{ACKNOWLEDGEMENTS}

Author would like to thank supervisors, dean; school of public health, Mount Kenya university and director institute of capacity development, Amref health Africa, for their advice, and support on this proposal. Their words of encouragements, motivation and knowledge positively impacted on the competition of this $\mathrm{PhD}$ research proposal.

Funding: No funding sources

Conflict of interest: None declared

Ethical approval: Not required

\section{REFERENCES}

1. Definition and classification of CKD. In: Kidney International Supplements. Elsevier Inc; 2013;3:1962.

2. Johnson C, Levey A, Coresh J, Levin A, Lau J, Eknoyan G. Clinical practice guidelines for chronic kidney disease in adults: part I definition, disease stages, evaluation, treatment, and risk factors. Am Fam Physician. 2004;70(5):869-76.

3. Singh S, Sharma R, Kumari M, Tiwari S. Insulin receptors in the kidneys in health and disease. World J Nephrol. 2019;8(1):11-22.

4. Mousa I, Ataba R, Al-ali K, Alkaiyat A, Zyoud SH. Dialysis-related factors affecting self-efficacy and quality of life in patients on haemodialysis: a crosssectional study from Palestine. Ren Replace Ther. 2018;4(1):1-12.

5. Wang H, Naghavi M, Allen C. Global, regional, and national life expectancy, all-cause mortality, and cause-specific mortality for 249 causes of death, 1980-2015: a systematic analysis for the Global Burden of Disease Study 2015. Lancet. 2016;388(10053):1459-544.

6. Kassebaum NJ, Arora M, Barber RM. Global, regional, and national disability-adjusted life-years (DALYs) for 315 diseases and injuries and healthy 
life expectancy (HALE), 1990-2015: a systematic analysis for the Global Burden of Disease Study 2015. Lancet. 2016;388(10053):1603-58.

7. Perico N, Remuzzi G. Chronic kidney disease in subSaharan Africa: A public health priority. Lancet Glob Heal. 2014;2(3):124-5.

8. Dwyer-Lindgren L, Cork MA, Sligar A. Mapping HIV prevalence in sub-Saharan Africa between 2000 and 2017. Nature. 2019;570(7760):189-93.

9. George C, Mogueo A, Okpechi I, Echouffo-Tcheugui JB, Kengne AP. Chronic kidney disease in lowincome to middle-income countries: The case $\mathrm{f}$ increased screening. BMJ Glob Heal. 2017;2(2):1-10.

10. Naicker S, Ashuntantang G. End Stage Renal Disease in Sub-Saharan Africa. Chronic Kidney Dis Disadvantaged Popul. Published online. 2017;12537.

11. Kaze AD, Ilori T, Jaar BG, Echouffo-Tcheugui JB. Burden of chronic kidney disease on the African continent: A systematic review and meta-analysis. BMC Nephrol. 2018;19(1):1-11.

12. Kalyesubula R, Nankabirwa JI, Ssinabulya I, et al. Kidney disease in Uganda: a community based study. BMC Nephrol. 2017;18(1):1-9.

13. Mugo P, Onsomu E, Munga B. An Assessment of Healthcare Delivery in Kenya under the Devolved System An Assessment Healthcare Delivery. 2018;19.

14. Mwangi. Kenya-Medical Equipment. export.gov. Available at: https://www.export.gov/article?id= Kenya-medical-equipment. Published 2019. Accessed on September 29, 2019.

15. Beerappa H, Chandrababu R. Adherence to dietary and fluid restrictions among patients undergoing hemodialysis: An observational study. Clin Epidemiol Glob Heal. 2019;7(1):127-30.

16. Karimi M, Brazier J. Health, Health-Related Quality of Life, and Quality of Life: What is the Difference?
Pharmacoeconomics. 2016;34(7):645-9.

17. Chege P. Multiple cardiovascular disease risk factors in rural Kenya: evidence from a health and demographic surveillance system using the WHO STEP-wise approach to chronic disease risk factor surveillance. South African Fam Pract. 2016;58(2):54-61.

18. Oluyombo R, Ayodele OE, Akinwusi PO. Awareness, knowledge and perception of chronic kidney disease in a rural community of South-West Nigeria. Niger J Clin Pract. 2016;19(2):161-9.

19. Wileman V, Farrington K, Wellsted D, Almond M, Davenport A, Chilcot J. Medication beliefs are associated with phosphate binder non-adherence in hyperphosphatemic haemodialysis patients. $\mathrm{Br} \mathrm{J}$ Health Psychol. 2015;20(3):563-78.

20. WHO. eHealth at WHO. WHO. Published online 2018. Available at: https://www.who.int/ehealth/ about/en/Accessed on October 23, 2019.

21. Singh, Panjwani, Puri, Delhi N. mHealth : Meaning, Purpose and Outcomes. Int $\mathbf{J}$ Comput Sci Inf Technol. 2016;7(5):2216-21.

22. Lingala SM, Ghany MGMMhs. Mobile Health Devices as Tools for Worldwide Cardiovascular Risk Reduction and Disease Management. 2017;25(3):289-313.

23. Kariuki EG. Adoption of m-health and usability challenges in m-health applications in kenya: case of uzazi poa m-health prototype application. J Econ Financ. 2016;3(1):56.

24. WHO. Image based information access for mobile phones. Proc-Int Work Content-Based Multimed Index. 2011;3:130-5.

Cite this article as: Josphat MM, Kariuki J, Kimathi G. mHealth intervention and health-related quality of life for haemodialysis patients in selected counties of Kenya. Int J Community Med Public Health 2022;9:1565-8. 